Revista Eletrônica em Gestão, Educação e Tecnologia Ambiental

Santa Maria, v. 19, n. 3, set-dez. 2015, p. 36-53

Revista do Centro de Ciências Naturais e Exatas - UFSM

ISSN : 22361170

\title{
As regras e a função reação da política monetária nos bancos centrais dos EUA, do Japão e da União Europeia
}

\author{
Monetary Policy Rules and reaction function in the Central Banks of the USA, \\ Japan and European Union \\ Romário Gollo Souza ${ }^{1}$, Divanildo Triches ${ }^{2}$ \\ Universidade de Caxias do Sul, RS \\ Universidade do Vale do Rio dos Sinos, RS.
}

\section{RESUMO}

Este artigo tem o objetivo de investigar a função de reação de política monetária, seguindo a abordagem da Regra de Taylor, para avaliar o desempenho da política monetária conduzida pela Reserva Federal (FED), pelo Banco do Japão (BOJ) e pelo Banco Central Europeu (ECB), durante o período de 1990 a 2008 para BOJ, para o ECB, em virtude da constituição da Área do euro; a análise abrange janeiro de 1998 a junho de 2008.Procedeu-se à realização das estimações por intermédio da aplicação do Método Generalizado dos Momentos (GMM), do Método dos Mínimos Quadrados Ordinários (OLS) e do Método da Máxima Verossimilhança (ARCH), cujos resultados sugerem que o Banco Central dos Estados Unidos, do Japão e da Área do euro leva em consideração nas suas decisões de política monetária, a evolução dos desvios ponderados das expectativas de inflação em relação à meta do presente ano e do ano seguinte, do hiato do produto e das taxas de câmbio. Além disso, encontrou-se para a economia japonesa uma função de reação de política monetária, em que a taxa de juro objetivo se ajusta, principalmente, de modo a estabilizar a inflação. Já, para os Estados Unidos e para a Área do euro, encontrou-se uma função de reação de política monetária na qual a taxa objetivo se move para acomodar as mudanças na inflação e com menor ênfase nas variações do hiato do produto.

Palavras-chave: Regras de política monetária, Função de reação, bancos centrais.

\section{ABSTRACT}

This paper investigates the function of reaction of monetary policy following the approach of the Taylor Rule to evaluate the performance of this policy, lead for the Federal Reserve (FED), for the Bank of Japan (BOJ) and for European Central Bank $(E C B)$, during the period selected for the research. It was considered for the analysis, as much for the FED how much for the BOJ, the period of January 1990 until June 2008; whereas for the ECB, in virtue of the constitution of the Euro Area, the analysis encloses January 1998 until June 2008. The estimates are made through the application of the Method of the Generalized Moments, of the Method of the Ordinary Least Squared and the Model of Autoregressive Conditional Heterocedasticity, whose results suggest that the Central banks of the U.S.A., the Japan and the Euro Area takes account its monetary policy decisions in the weighed deviation of the expectations of inflation, the output gap and the exchange rate. Moreover, a function of reaction of monetary policy for the Japanese economy shows that the objective real interest rate is adjusted in order to stabilize the inflation mainly. A function of reaction of monetary policy for the U.S.A. and the Euro area points out the target interest rate is set to accommodate the changes in the inflation and also, but with minor intensity, the output gap

Keywords: Monetary Policy Rules, Reaction Function, Central Banks.

JEL Classification: H62, H63, E63 


\section{INTRODUÇÃO}

A discussão sobre quais são os objetivos que a política monetária deve perseguir e como deve ser conduzida tem recebido a atenção de diversos estudiosos, ao longo da história da economia monetária. Até o início da década de 70, a política monetária era entendida como um instrumento de política econômica e, como tal, deveria ser utilizado pelos bancos centrais, tendo como principal finalidade a sustentação de elevados níveis de emprego. Entretanto, por volta dos anos 80, esse objetivo foi direcionado para a busca da estabilidade dos preços.

Ao longo da segunda metade do século XX, ressalta-se o debate entre keynesianos e monetaristas quanto à influência da moeda sobre variáveis nominais e reais, a curto e longo prazo e a necessidade ou não de regras fixas na condução da política, principalmente, entre Friedman (1968) e Tobin (1987). Além disso, são abordados na literatura a neutralidade da moeda, inclusive no curto prazo como em Lucas (1981) e Barro (1976); o viés inflacionário de políticas discricionárias e a questão da credibilidade com ênfase na inconsistência dinâmica como em Kydland e Prescott (1977) e Barro e Gordon (1983). As discussões recentes focam as propostas de independência do banco central, como Cukierman (1992) e Alesina; Summers, (1993); os contratos ótimos e regras contingentes, em Rogoff (1985), Lohmann (1992); Walsh (1995) e Taylor (1999); e as metas de inflação em Bernanke; Mishkin (1997) e Bernanke et al. (1999).

A política monetária, ao fazer uso do instrumento da taxa de juros, para controlar os níveis de inflação e produto, interfere no comportamento dos agentes econômicos, que ganham ou perdem seus recursos com as flutuações dessas taxas. Consequentemente, os mercados financeiros estão sempre em busca de prever os ajustes da política monetária. Assim, essa interação - os formuladores de política monetária tentando compreender e interpretar os mercados e vice-versa - torna a tarefa de projetar uma regra ótima de política monetária num problema bastante complexo.

Sabe-se, contudo, que, no longo prazo, a inflação é um fenômeno estritamente monetário e que depende do controle da emissão monetária por parte do banco central. Porém, no curto prazo, debate-se na literatura que a reação ótima da política monetária, diante de choques sobre a economia, pode envolver a combinação de alguma regra de juros com algum grau de discricionariedade e estabelecimento de metas de inflação. Destaca-se que há um vasto debate na literatura econômica, que faz referência à questão; regra versus discricionariedade. ${ }^{1}$

As vantagens de um consenso de política monetária são largamente dependentes do entendimento e da previsibilidade dessas políticas pelo setor privado. Embora tenha havido um aperfeiçoamento nessa área, ainda discute-se de que forma os bancos centrais conduzem a política monetária, para aumentar o produto e reduzir o desemprego, sem causar mais inflação. Para isso, torna-se importante também analisar o desenvolvimento e o gerenciamento dos sistemas de pagamentos dos bancos centrais, que, aliados à condução

1 Para uma abordagem detalhada dessa discussão, vejam-se Simons (1936), Kydland e Prescott (1977), Barro e Gordon (1983), Argy (1988), Fischer (1990), Taylor (1993), Rogoff (1995), Ball (1999), Bernanke et al. (1999), Orphanides (2003) e Woodford (2003). 
eficaz da política monetária, procuram essencialmente a redução dos riscos envolvidos nas transações e buscam evitar o colapso do sistema financeiro como um todo.

Nesse sentido, este artigo tem o objetivo de investigar a função de reação de política monetária, seguindo a abordagem da Regra de Taylor, para avaliar o desempenho da política monetária conduzida pela Reserva Federal (FED), pelo Banco do Japão (BOJ) e pelo Banco Central Europeu (ECB), durante o período de 1990 a 2008. Para tanto, além dessa introdução, na seção 2, encontra-se uma breve revisão das regras e a discricionariedade na política monetária. A estimação da função-reação da política monetária dos países selecionados, na especificação de Taylor (1993), é desenvolvida na seção 3. A análise dos resultados das estimativas é encontrada 4 . Por fim, a seção 5 apresenta as conclusões.

\section{As regras e a discricionariedade na política monetária}

O uso de regras pelos bancos centrais está amplamente associado ao conceito de credibilidade, que pode ser entendido como o nível de confiança que os agentes econômicos têm no cumprimento de uma determinada política previamente anunciada. Assim, uma política irá transmitir maior credibilidade, se ela sinalizar aos agentes uma baixa probabilidade da ocorrência de inconsistência temporal.

Nesse sentido, Poole (1999) e Blanchard (1999) abordam que a inconsistência temporal descreve os incentivos dos formuladores de política em desviar-se de uma política, após ela ter sido anunciada e os tomadores de decisão privados terem reagido a ela. Em outras palavras, a inconsistência dinâmica ocorre quando uma decisão de política, que faz parte de um plano ótimo formulado numa data inicial, deixa de ser ótima sob o ponto de vista de uma data futura, mesmo quando não há nenhuma nova informação ou alteração na estrutura de preferências. Esse fato acaba gerando um viés inflacionário sob uma política monetária discricionária. Greenspan (2004) e Orphanides (2007) argumentam que a política sistemática, isto é, baseada em um plano de contingência ou regra, tem vantagens não despresíveis sobre uma política puramente discricionária. Ao se comprometer em perseguir uma regra, os formuladores de política monetária podem evitar a ineficiência associada ao problema da inconsistência temporal, que aumenta quando a política é formulada de uma maneira discricionária. Dessa forma, seguir uma regra permite que os responsáveis políticos comuniquem e expliquem suas ações de política mais efetivamente. Assim, políticas baseadas em uma regra bem-compreendida realçam a responsabilidade do banco central e melhoram a credibilidade das futuras ações políticas.

O uso de uma regra pode remover o viés inflacionário e estabilizar de forma simultânea a inflação e o produto como mostra Taylor (1993). A regra proposta por esse autor permite o uso da política monetária quando há desvios em relação às metas de inflação e de produto da economia. A regra de Taylor foi desenvolvida para permitir que FED possa ajustar as taxas de juros de curto prazo, para reduzir a volatilidade macroeconômica. A persistência da estabilidade do nível geral de preços, como objetivo prioritário evita a perda da credibilidade pela autoridade monetária. 
De acordo com Taylor (1993), o comportamento das taxas de juros, $i_{t}$, poderia ser representado por uma relação linear com a taxa de inflação, $\pi_{t}$, uma taxa de juros de equilíbrio, $r^{*}$, mais uma ponderação entre dois desvios: a) a diferença entre taxa de inflação (medida pelo deflator do PIB) e a meta de inflação $\pi_{t}^{*}$; b) o desvio percentual do PIB potencial $y_{t}$. Dessa forma, a Regra de Taylor depende de duas variáveis exógenas ao modelo, que são a meta de inflação e o PIB potencial. A relação dessas variáveis pode ser visualizada conforme a equação (1):

$$
i_{t}=\pi_{t}+r^{*}+0,5\left(\pi_{t}-\pi^{*}\right)+0,5\left(y_{t}\right)
$$

Taylor (1993) assumiu que as ponderações da equação (1) foram utilizadas pelo FED em relação aos desvios da inflação e o PIB e seriam de 0,5. Assim se, por exemplo, a inflação fosse um ponto percentual acima da meta, o FED deveria elevar em $0,5 \%$ as taxas de juros. A taxa de juros e a meta de inflação de equilíbrio foram assumidas em $2 \%$. As regras de política monetária, como a de Taylor, são simples e reativas (funções de reação), e ajustam o instrumento político da taxa de juros em resposta à evolução da taxa de inflação e da atividade econômica.

A partir do trabalho de Taylor (1993), Clarida et al. (1998) estimaram uma função de reação para os principais bancos centrais, no período de abril/1979 a dezembro/1994. Os bancos centrais foram separados em dois grupos: a) G3, constituído pelas autoridades monetárias da Alemanha, do Japão e dos EUA e b) E3, formado pelos bancos centrais do Reino Unido, da França e Itália. A função de reação proposta, em que as variáveis da função são os desvios da inflação e do hiato do produto em relação a seus valores-meta, consistiu numa estrutura forward-looking. Nesse caso, a política monetária tenderia a afetar contemporaneamente a inflação, mesmo na ausência de efeitos cambiais, pois a expectativa de aumento do hiato de produto, no período seguinte, influencia negativamente a inflação no período atual.

Clarida et al. (1998) encontraram que, desde 1979, cada banco central do G3 tem perseguido uma forma implícita de meta inflacionária, para a qual é possível considerar o amplo sucesso da política monetária, naqueles países, durante o período. A análise das evidências apontadas também sugere que os bancos centrais do G3 reagem à inflação antecipada ao invés da inflação passada. Para os países do grupo E3, as autoridades monetárias foram fortemente influenciadas pela política monetária alemã.

Para estimar a função de reação de política monetária do Banco do Japão, no período de abril/1979 a dezembro/1994, Andrade e Divino (2005) assumiram vários pressupostos como: (i) a taxa oficial de desconto é o principal instrumento do banco central; (ii) os salários e preços apresentam viscosidade - há rigidez nominal no curto-prazo; (iii) as autoridades monetárias seguem metas reais (estabilidade da produção, estabilidade da taxa de câmbio real) e metas nominais (estabilidade dos preços). Dessa forma, a função de reação de política monetária pode ser descrita pela equação (2):

$$
i_{t}^{*}=\bar{i}+\beta\left(\mathrm{E}\left[\pi_{t} \mid \Omega_{t}\right]-\pi^{*}\right)+\gamma\left(\mathrm{E}\left[h_{t} \mid \Omega_{t}\right]-h_{t}^{*}\right)+\xi\left(\mathrm{E}\left[e_{t} \mid \Omega_{t}\right]-e_{t}^{*}\right)
$$


Assim, os bancos centrais têm um objetivo para a taxa de juros nominal de curtoprazo, $i_{t}^{*}$, estabelecida num determinado estado da economia. No caso dos parâmetros, os autores assumem que o objetivo depende das expectativas sobre a inflação, o produto e a taxa de câmbio. Assim, $\bar{i}$ é a taxa de juros nominal de equilíbrio de longo prazo; E é o valor esperado da respectiva variável, condicionado ao cenário de informações avaliadas pela autoridade monetária no início do período $t\left(\Omega_{t}\right)$. A função supõe, ainda, três metas alternativas com relação à de inflação, $\pi^{*}$, ao produto potencial, $h_{t}^{*}$, e a taxa de câmbio, $e_{t}^{*}$.

Essa formulação permite que a autoridade monetária direcione a inflação, o hiato do produto e a taxa de câmbio esperados. Nesse contexto, Clarida et al. (1998), justificaram que uma simples regra como a descrita na equação (2) não pode capturar a tendência dos bancos centrais em suavizar mudanças nas taxas de juros. Diante dessa dificuldade, Andrade e Divino (2005) consideraram que a taxa de juros atual, parcialmente ajustada ao objetivo, é dada pela equação (3):

$$
i_{t}=(1-\rho) i_{t}^{*}+\rho i_{t-1}+v_{t}
$$

onde o parâmetro, $\rho \in[0,1]$, captura o grau de suavização da taxa de juros. A especificação também inclui um choque aleatório exógeno na taxa de juros, $v_{t}$. Algumas interpretações possíveis em que $v_{t}$ pode refletir um componente aleatório puro para a política, ou pode aparecer porque os bancos centrais preveem de forma imperfeita a demanda por reservas e, por algumas razões, não repõem instantaneamente a oferta de reservas para contrabalancear os choques. Combinando o modelo objetivo, dado pela equação (2), com o mecanismo de ajuste parcial (3), tem-se a equação (4):

$$
i_{t}=(1-\rho)\left\{\alpha+\beta\left(\mathrm{E}\left[\pi_{t} \mid \Omega_{t}\right]-\pi_{t}^{*}\right)+\gamma\left(\mathrm{E}\left[h_{t} \mid \Omega_{t}\right]-h_{t}^{*}\right)+\xi\left(\mathrm{E}\left[e_{t} \mid \Omega_{t}\right]-e_{t}^{*}\right)\right\}+\rho i_{t-1}+v_{t}
$$

onde $\alpha \equiv \bar{i}-\beta \pi^{*}$. Assim, a solução estática de longo prazo da equação (4) pode ser escrita conforme a equação (5):

$$
i=\alpha+\beta \pi+\gamma h+\xi e^{\prime}+\varepsilon
$$

onde $i=i_{t}=i_{t-1}, e^{\prime}=e_{t}-e_{t}^{*}$ e $v_{t} /(1-\rho)$. Para o caso em que $\beta>1$ e $\gamma>0$, a taxa de juro real objetivo se ajusta de modo a estabilizar a inflação e o produto. Contudo, se $\beta<1$, a taxa real objetivo se move para acomodar as mudanças na inflação. Um valor pequeno de $\beta$ significa que a autoridade monetária aumenta a oferta de liquidez, de forma suficiente para moderar a elevação da taxa de juros, o que induz também um acréscimo na taxa de inflação. Esse pode ser o caso em a variação da taxa de juro nominal não é suficiente para provocar uma variação no mesmo sentido da taxa de juro real. A ideia equivalente se aplica em relação ao parâmetro $\gamma$ o qual deverá ser não negativo para a regra ser estabilizadora. Por último, as estimações e as funções de reação de política monetária podem ser realizadas sob três formas, ou seja, 
Método Generalizado dos Momentos, Método dos Mínimos Quadrados Ordinários e Método da Máxima Verossimilhança.

\section{Estimação da função-reação da política monetária}

\subsection{Definições de variáveis}

A taxa de juros tem sido reconhecida como o instrumento principal da política monetária da maior parte dos bancos centrais, para alcançar a estabilidade da inflação e do produto, e talvez a estabilidade da taxa de câmbio. Diante disso, buscou-se identificar a função de reação política monetária conduzida pela Reserva Federal (FED), e pelo Banco do Japão (BOJ) no período de janeiro/1990 até junho/2008 e pelo Banco Central Europeu (ECB) ao longo de janeiro/1998 a junho/2008. ${ }^{2}$

Para estimar uma função de reação de política monetária para cada país, os EUA, o Japão e a Área do Euro, foi usada a equação (5), e as variáveis consideradas foram as seguintes: i) $\pi$ é a taxa de inflação, medida pela variação do Índice de Preços ao Consumidor (IPC). Para dados mensais, a variação do IPC foi acumulada durante os últimos seis e doze meses e definida por $\pi(6)_{t}$ e $\pi(12)_{t}$, respectivamente. Todas as séries do IPC foram obtidas do Instituto de Pesquisa Econômica Aplicada (Ipea); ii) A variável $h_{t}$ é o hiato do produto, que corresponde ao desvio, do índice da produção industrial de uma tendência ao quadrado. Para construir o índice e obter a série do desvio foi utilizado o filtro HodrickPrescott (HP). A série temporal mensal foi obtida, para os EUA, nas estatísticas do Board of Governors of the Federal Reserve System; para o Japão, nas estatísticas do Bank of Japan e para a Área do euro, na agência oficial de estatística da Comunidade Europeia - Eurostat; iii) A variável $e_{t}$ é o desvio da taxa de câmbio real, que corresponde ao desvio da taxa de câmbio em relação ao seu valor de paridade (PPP). Para construir o índice e obter a série do desvio, também foi utilizado o filtro Hodrick-Prescott (HP). A série foi obtida no Ipea; iv) Por fim, a variável $i_{t}$ é dada pela taxa de juros básica de curto prazo de cada país, acumulada nos últimos seis meses. Para os EUA, a taxa é representada pelos federal funds; para o Japão, utilizou-se a taxa call rate e, para a Área do euro, foi adotada a refi rate. Em ambos os países, as séries de juros foram obtidas no banco de dados do Ipea. Todas as variáveis são medidas sob forma logarítmica.

\subsection{Testes de Raiz Unitária}

Inicialmente foram realizados os testes de raiz unitária com por meio do teste DickeyFuller Aumentado (ADF), que permitiu concluir que, em nível, a série $e_{t}$ é estacionária para todos os países. ${ }^{3}$ As séries temporais $h_{t}$ e $i_{t}$ são estacionárias apenas nos EUA. Para as variáveis $\pi(6)_{t}$ e $\pi(12)_{t}$ rejeita-se a hipótese de elas são integradas de ordem zero para todos os casos investigados, ou seja, EUA, JAPÃO e Área do euro. Já em primeira diferença, todas as variáveis são estacionárias, exceto a variável $i_{t}$ na Área do euro.

\footnotetext{
2 Segue-se a abordagem de Taylor (1993) com as modificações proposta por Clarida et al. (1998) e após por Andrade e Divino (2005).

${ }^{3}$ Os testes ADF e NG foram efetuados por meio do software Eviews 5.0, os quais estão reportados nas tabelas 1 a 4 no Anexo.
} 
O teste Ng Perron, em nível e em primeira diferença, para as séries temporais dos EUA, considerando a presença de tendência e a presença de constante e tendência, indica que as variáveis $e_{t}$ e $h_{t}$ são estacionárias. As variáveis $\pi(6)_{t}$ e $\pi(12)_{t}$ são integradas de ordem I(1), em nível e em primeira diferença, com tendência; e, quando se considerada a presença de constante e tendência, a variável $\pi(6)_{t}$ continua sendo integrada de ordem I(1), enquanto que a variável $\pi(12)_{t}$ passa a ser estacionária. Para a variável $i_{t}$, aceita-se a hipótese de raiz unitária apenas quando o teste é aplicado em nível, com tendência. Nos demais casos, essa série é integrada na ordem zero. O teste $\mathrm{Ng}$ Perron, quando aplicado para as séries da economia japonesa, indica que a variável $e_{t}$ é estacionária em todos os casos, exceto em primeira diferença, com constante. As variáveis $h_{t}$ e $i_{t}$ apresentam raiz unitária. Já as variáveis $\pi(6)_{t}$ e $\pi(12)_{t}$ sempre são I(1) em nível. Contudo, em primeira diferença, a variável IPC6 é estacionária com constante e tendência. A série $\pi(12)_{t}$ é estacionária quando se considera apenas a tendência.

A avaliação dos resultados do teste $\mathrm{Ng}$ Perron para as variáveis da Área do euro, permite aceitar a hipótese de estacionariedade da $e_{t}$ é integrada de ordem um apenas em nível, considerando a presença de constante e tendência. A variável $h_{t}$ apresenta raiz unitária em nível, porém em primeira diferença se torna $\mathrm{I}(0)$. As variáveis $\pi(6)_{t}$ e $\pi(12)_{t}$ são I(1) em nível, porém em primeira diferença, a variável $\pi(12)_{t}$ se torna estacionária. A variável $i_{t}$ é estacionária sob todas as variações do teste.

Diante desses resultados, não houve possibilidade de aplicação de testes de cointegração entre as variáveis. Assim, tomando por base a equação (5), estimaram-se diversos modelos através da aplicação do Método Generalizado dos Momentos (GMM) do Método dos Mínimos Quadrados Ordinários (OLS) e do Método da Máxima Verossimilhança (ARCH). Inclui-se uma variável dummy na equação (5), para captar o comportamento da taxa de juros de curto prazo de cada país, ao longo do período analisado. Os coeficientes encontrados para os modelos, com ou sem a presença da variável dummy, são comparados entre si, a fim de se encontrar a melhor função de reação de política monetária para cada um dos países.

\section{Análise dos resultados das estimativas}

O modelo descrito previamente pelas equações (5) foi aplicado para os EUA, o Japão e a Área do euro, sem e com a inclusão da variável dummy de juros. Os resultados encontrados para a função de reação de política monetária de cada país estão apresentados nas equações (6) a 8. O número entre parênteses representa o desvio padrão de cada coeficiente estimado. Os critérios de avaliação considerados para escolha dos modelos são: os Critérios de Informação Akaike (AIC), os Critérios Schwartz (SC), Erro Absoluto Médio (MAE), Erro Percentual Absoluto Médio (MAPE), Raiz do Erro Quadrático Médio (RMSE) e Coeficiente de Desigualdade Theil (TIC). Quanto menores os valores dos critérios AIC, SC, RMSE, MAE e MAPE, mais adequado é o modelo. O inverso ocorre para o critério TIC. 
Os resultados econométricos para a série de dados mensal nos EUA, sem a inclusão da variável dummy de juros no modelo, apontam coeficientes estatisticamente significativos para o modelo quando esse é estimado pelos métodos OLS e ARCH, tanto para o modelo que utiliza a variável $\pi(6)_{t}$ como para o modelo estimado com a variável $\pi(12)_{t}$. Porém, há presença de heteroscedasticidade em ambos as especificações, quando é usado o primeiro método. Dessa forma, os resultados obtidos tendem a ser mais adequados com o emprego do método $\mathrm{ARCH}$, com a especificação da variável $\pi(12)_{t}$, pois se pode considerar que os coeficientes são estatisticamente significativos, e que a heteroscedasticidade foi corrigida a um nível de confiança de $1 \%$. Os critérios AIC e SC permitem inferir que o modelo com $\pi(12)_{t}$ é o melhor ajustado do que aquele que especifica $\pi(6)_{t}$ com variável explicativa.

Após a definição do modelo e o método pelo qual será estimado, avalia-se a necessidade ou não de uma variável dummy. Para isso, procede-se a uma análise comparativa entre as taxas de juros de curto prazo efetivas e estimadas pelas funções de reação de política monetária, definidas pelos métodos $\mathrm{ARCH}$ nas duas alternativas dos Estados Unidos no período de janeiro de 1990 a junho de 2008, conforme é mostrado na figura 1. Pela análise dessa figura e pelos critérios RMSE, MAE, MAPE, e TIC, é possível inferir que a função de reação de política monetária estimada com melhores ajustes é aquela que incorpora as variáveis $\pi(12)_{t}$ e uma dummy de juros como explicativas. Essa especificação é expressa pela equação (6). Nota-se que os coeficientes ou elasticidades estimadas, nesse caso, foram 0,99 para a inflação, $-0,13$ para o hiato do produto e 0,05 para o desvio taxa de câmbio. Tais resultados indicam que as autoridades monetárias tendem a responder mais rapidamente à mudança na taxa de inflação do que no hiato.

$$
\begin{aligned}
& i_{t}=\underset{(0,00)}{0,01 D}+\underset{(0,00)}{0,99 \pi(12)_{t}}-\underset{(0,02)}{0,13 h_{t}}+\underset{(0,01)}{0,05 e_{t}}+\varepsilon \\
& \text { onde } h_{t}=\underset{(0,00)}{0,0000000147+} \underset{(0,15)}{0,96 \varepsilon_{t-1}^{2}-0,76 \varepsilon_{t-2}^{2}}+\underset{(0,19)}{0,83} h_{t-1}
\end{aligned}
$$

Figura 1: Evolução comparativa das taxas de juros efetivos e estimados dos EUA de janeiro de 1990 até junho de 2008




Para a economia japonesa, os resultados mostram que os coeficientes são estatisticamente significativos apenas pelo método $\mathrm{ARCH}$, com a incorporação da variável CPI6 no modelo. Contudo, com a inclusão da variável dummy de juros, com variável independente, coeficientes passam a ser estatisticamente significativos apenas pelo método ARCH com a variável $\pi(12)_{t}$. Todas as demais especificações revelam coeficientes não significativos e heteroscedasticidade.

A figura 2 e os critérios RMSE, MAE, MAPE e TIC indicam que o método de estimação ARCH, e o modelo especificado com variável dummy de juros e a variável $\pi(12)_{t}$ representa a melhor função de reação de política monetária para a economia japonesa, a partir da década de 90 . Os parâmetros dessa função são reportados na equação (7). Nota-se nesse caso que a reação da taxa de juros, em relações à taxa de inflação, se dá na mesma proporção como foi encontrada para a economia norte-americana. Já a reação das autoridades monetárias, em relação ao hiato do produto e ao desvio de taxa de câmbio, é pouco expressiva, ou seja, uma elasticidade da ordem de -0,006 e -0,016, respectivamente. Esses resultados permitem inferir que as autoridades monetárias tendem a dar mais ênfase na estabilidade da taxa de inflação do que nos choques de taxa de câmbio real e de produto.

$$
\begin{aligned}
& i_{t}=\underset{(0,00)}{0,002 D}+\underset{(0,00)}{1,00 \pi(12)_{t}}-\underset{(0,003)}{0,006 h_{t}}-\underset{(0,007)}{0,016 e_{t}}+\varepsilon \\
& h_{t}=\underset{(0,00)}{0,00000224+\underset{(0,11)}{0,90} \varepsilon_{t-1}^{2}-0,67 \varepsilon_{t-2}^{2}+0,73 h_{t-1}}
\end{aligned}
$$

Figura 2: Evolução comparativa das taxas de juros efetivos e estimados da economia japonesa de janeiro de 1990 até junho de 2008

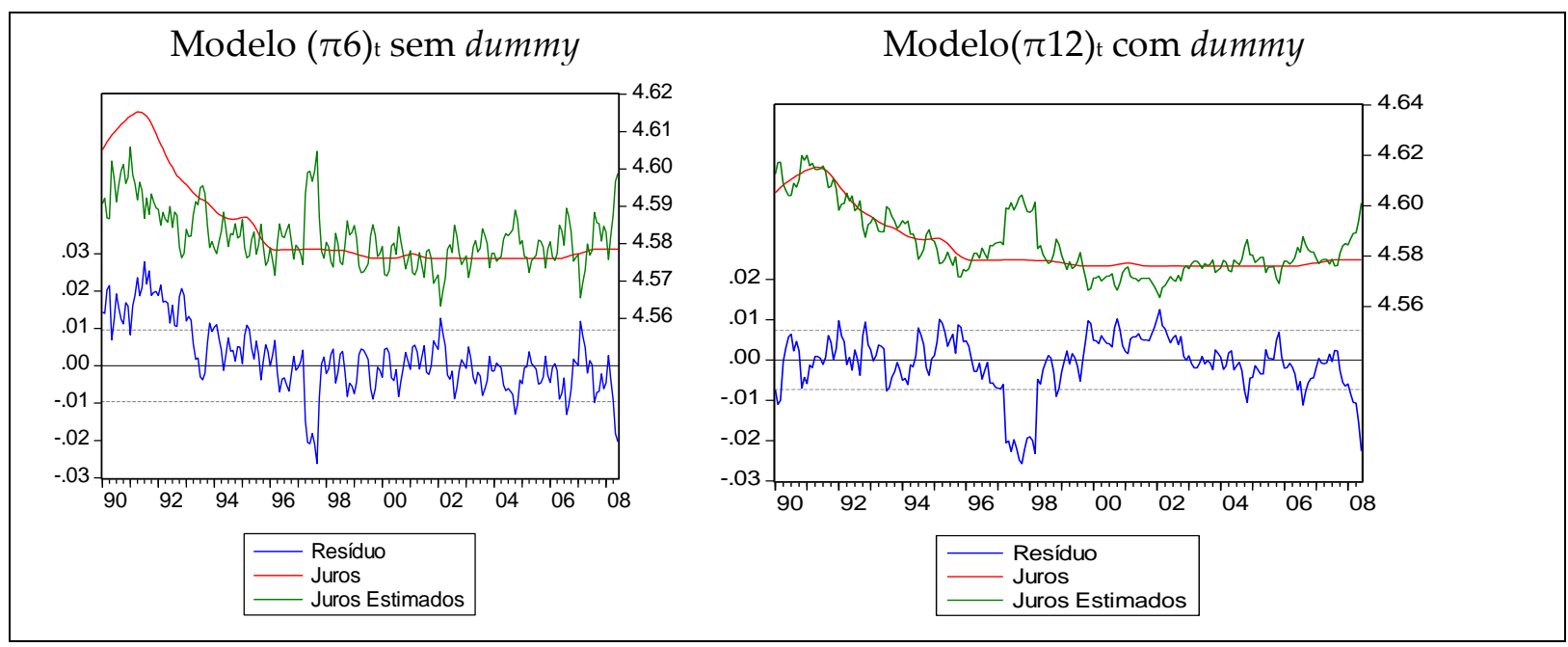

Os resultados econométricos, provenientes da estimação da função reação das autoridades monetária da Área do euro, indicaram que o modelo ARCH especificado tanto com a variável $\pi(6)_{t}$, como com a variável $\pi(12)_{t}$ revelam ausência de heteroscedasticidade . Os coeficientes de todas as variáveis do modelo ARCH com CPI12 são estatisticamente significativos; no entanto, no modelo ARCH com a variável CPI6, a constante e o coeficiente $h_{t-1}$, na equação de variância, não são significativos. Portanto, pela avaliação do desempenho do modelo, sem a inclusão da variável dummy de juros, está naqueles que inclui a variável 
$\pi(12)_{t}$. Da mesma forma como foi procedido para os casos anteriores, como nos EUA e no Japão, analisam-se os resultados comparando-os com a incorporação ou não de uma variável dummy . A figura 3 e os critérios RMSE, MAE, MAPE e TIC apontaram que o método de estimação $\mathrm{ARCH}$ e a especificação do modelo com variável dummy tendem a mostrar melhor aderência da estimação da função reação das autoridades monetárias da Área do euro.

A equação (8) mostra as elasticidades estimadas para cada uma das variáveis explicativas. A reação da taxa de juros, em relação à mudança da taxa de inflação, ocorre na mesma proporção e direção. O coeficiente estimado, como nos casos dos EUA e Japão, é igual à unidade. Para as variáveis desvio da taxa de câmbio real e hiato do produto, os coeficientes são ambos positivos e giram em torno de 0,10.

$$
\begin{aligned}
& i_{t}=\underset{(0,00)}{0,009 D}+\underset{(0,00)}{0,99 \pi(12)_{t}}+\underset{(0,03)}{0,12 h_{t}}+\underset{(0,02)}{0,10 e_{t}}+\varepsilon \\
& \text { Com } h_{t}=\underset{(0,00)}{0,00000341+0,90 \varepsilon_{t-1}^{2}} \underset{(0,27)}{0}
\end{aligned}
$$

Figura 3: Evolução comparativa das taxas de juros efetivos e estimados da economia da Área do euro de janeiro de 1998 até junho de 2008

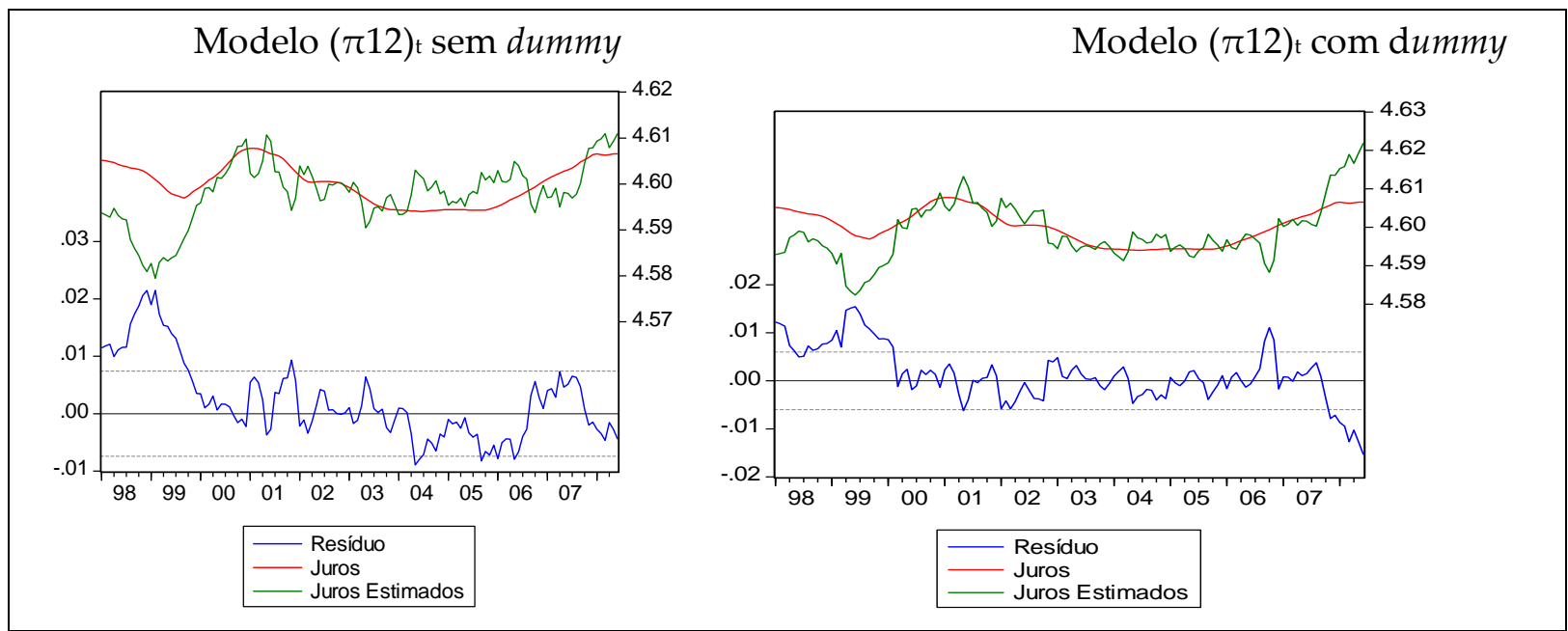

As evidências empíricas têm mostrado que as regras de Taylor descrevem razoavelmente bem a reação das principais autoridades monetárias, em especial o FED e o Bundesbank, ao longo das duas últimas décadas, em relação à mudança da taxa de inflação, crescimentos do produto e taxa de câmbio. Nesse contexto, parece razoável argumentar que uma regra de Taylor poderá constituir uma referência para o debate sobre política monetária. Em relação à análise dos parâmetros $\beta, \gamma$ e $\xi$, estimados nas equações (6) a (8), a tabela 1 apresenta uma síntese, para efeito ilustrativo, dos valores encontrados na literatura para a economia norte-americana, japonesa e para a Área do euro.

Observa-se, na tabela 1, que o parâmetro que mede o efeito da inflação sobre a taxa de juros varia de 1,2 a 3,0 nos Estados Unidos e de 0,78 a 2,68 no Japão e 1,3 a 2,0 na Alemanha e zona do euro. Já no que se refere ao hiato do produto, a resposta medida situa-se na faixa de $-0,05$ à unidade, para o caso da economia norte-americana, bem menor para a economia 
japonesa, isto é, os coeficientes giram entre 0,01 e 0,08 e, para a Alemanha e zona do euro o intervalo passa de 0,07 a 0,25 . Por último, a reação da taxa de juros de curto prazo, em relação ao desvio da taxa de câmbio real de equilíbrio para os poucos estudos realizados também é pouco significativa; os parâmetros variam na ordem de 0,07 nos Estados Unidos e 0,04 no Japão.

Tabela 1 - Síntese dos coeficientes estimados das variáveis desvio de inflação, hiato do produto e desvio da taxa de câmbio real

\begin{tabular}{|c|c|c|c|c|}
\hline País & Autor & $\begin{array}{c}\text { Desvio de } \\
\text { inflação }(\beta)\end{array}$ & $\begin{array}{c}\text { Hiato do } \\
\text { produto }(\gamma)\end{array}$ & $\begin{array}{c}\text { Taxa de } \\
\text { Câmbio } \\
(\xi)\end{array}$ \\
\hline EUA & Taylor (1993) & 1,50 & 0,50 & - \\
\hline EUA & Taylor (1999a) & 1,50 & 1,00 & - \\
\hline EUA & Ball (1997) & 1,50 & 1,00 & - \\
\hline EUA & Christiano (1999) & 3,00 & 0,50 & - \\
\hline EUA & Clarida, et al. (1998) & 1,80 & 0,12 & - \\
\hline EUA & $\begin{array}{l}\text { Rotemberg e Woodford } \\
\text { (1998) }\end{array}$ & 1,20 & 0,06 & - \\
\hline EUA & Chada, et al. (2004) & 1,25 & 0,71 & 0,07 \\
\hline EUA & Molodtsova et al. (2008) & 2,68 & $-0,05$ & - \\
\hline Japão & Clarida et al. (1998) & 2,04 & 0,08 & - \\
\hline Japão & Chada et al. (2004) & 1,11 & 0,01 & 0,002 \\
\hline Japão & Andrade e Divino (2005) & 0,78 & 0,07 & 0,04 \\
\hline Alemanha & Clarida et al. (1998) & 1,31 & 0,25 & - \\
\hline Alemanha & Peersman e Smets (1998) & 1,30 & 0,28 & - \\
\hline Área do euro & Molodtsova et al. (2008) & 2,09 & 0,07 & - \\
\hline
\end{tabular}

Fonte: Chada et al. (2004) e Molodtsova et al. (2008)

Por fim, destaca-se que, para o caso em que $\beta>1$ e $\gamma>0$, a taxa de juro real objetivo se ajusta de modo a estabilizar a inflação e o produto, como foi abordado previamente. Esse fato é observado na literatura empírica para a economia japonesa, alemã e da zona do euro. Esses resultados são suportados pelas estimativas encontradas no presente estudo para a zona do euro. Já para economia dos Estados Unidos, os resultados obtidos são próximos daqueles estimados por Molodtsova et al. (2008). Em relação ao parâmetro $\xi$, tanto a literatura como os resultados mostram que é pouco significativo, o que revela que os bancos centrais de economias industrializadas tendem a não reagir como resposta à mudança no desvio da taxa de câmbio, ou apenas muito lentamente.

\section{Conclusões}

Atualmente, os bancos centrais concentram-se em realizar intervenções que buscam controlar as taxas de juros de curto prazo no mercado interbancário. Diante desse fato, FED, o BOJ e o ECB levam em consideração um conjunto de fatores na determinação das taxas de juros, com propósito de convergir as expectativas de inflação para uma meta desejada. Esse 
procedimento é dado a partir de uma função que minimize desvios do produto e da inflação em relação às suas metas.

O Japão, assim como os EUA e a Área do euro, também se encontra atualmente entre os países que não adotam de forma explícita, o regime de metas de inflação. A partir de 2006, o BOJ adotou uma política monetária mais restritiva e alterou, em março de 2006, o arranjo para a condução da política monetária: a meta operacional voltou a ser a taxa de juros básica, e o excesso de liquidez no saldo em conta corrente começou a ser reduzido.

O regime de política monetária da Área do euro não corresponde exatamente a um regime de metas de inflação, embora exista um teto explícito para o conceito de estabilidade de preços. Tampouco se trata de um regime de metas monetárias, apesar de ser definido um valor de referência para a expansão monetária. A estratégia intermediária adotada confere flexibilidade à política monetária, característica desejável, considerando-se sua aplicação por um banco central novo, atuando em uma área monetária recém-criada, requerendo, portanto, um processo de sintonia fina.

Os resultados permitem concluir que, para a zona do euro, a taxa de juro real objetivo se ajusta de modo a estabilizar a inflação e o produto. Já para os EUA e para a economia japonesa, encontrou-se uma função de reação de política monetária na qual a taxa real objetiva se move para acomodar as mudanças na inflação. Além disso, a variação da taxa de juro nominal não é suficiente para provocar uma variação no mesmo sentido da taxa de juro real. Ideia semelhante se aplica em relação ao parâmetro $\gamma$, que deverá ser não negativo para a regra ser estabilizadora, o contrário do que foi encontrado na função de reação para os EUA e para a Área do euro.

Os bancos centrais de economias industrializadas, como dos EUA, do Japão e da Área do euro tendem a ter baixa reação à resposta à mudança no desvio da taxa de câmbio. As respostas ao câmbio aumentam o bem-estar em uma economia aberta, pois, como há rigidez nominal dos preços dos bens produzidos domesticamente e dos preços domésticos das importações, variações no câmbio nominal não são imediatamente repassadas para a inflação e o hiato do produto. As respostas adequadas da política monetária ao desvio da taxa de câmbio e à desvalorização nominal têm, portanto, efeitos adicionais nas expectativas e nas decisões de preços e produção de um setor privado.

A evidência empírica tem mostrado que as regras de Taylor descrevem razoavelmente bem o comportamento das principais autoridades monetárias, em especial, o FED, ao longo das duas últimas décadas - um período em que a atuação da política monetária foi considerada bem-sucedida na redução da inflação. Nesse contexto, parece razoável argumentar que uma regra de Taylor poderá constituir uma referência para o debate sobre política monetária. 
ALESINA, A; SUMMERS, L. Central bank independence and macroeconomic performance: some comparative evidence. Journal of Money, Credit and Banking, v. 25, n. 2, p. 151-62, May 1993.

ANDRADE, J.P; DIVINO, J. A. Monetary policy of the Bank of Japan - inflation target versus exchange rate target. Japan and the world economy, v.17, p. 189-208, 2005.

ARGY, V.A. Post-War History of the Rules versus Discretion Debate. BNL quarterly review, v.12, p.147-177, 1988.

BARRO, R. Rational expectations and the role of monetary policy (1976). Reimpresso em: LUCAS, R; SARGENT, T. (Ed.). Rational Expectations and Econometric Practice. Minneapolis: University of Minnesota Press, 1981.

BARRO, R. J.; GORDON, D. Rules. discretion and reputation in a model of monetary policy. Journal of Monetary Economics, v. 12. p. 101-121, North-Holland, 1983.

BERNANKE, B; MISHKIN, F. Inflation targeting: a new framework for monetary policy? Journal of Economic Perspectives, v. 11, n. 2, Spring 1997.

BERNANKE, B., LAUBACH, T., MISHKIN, F., POSEN, A. Inflation targeting: lessons from the international experience. Princeton, Princeton University Press, 1999.

CALVO, G.; REINHART, C. Fear of Floating. Quarterly Journal of Economics, v. 17. n. 2, May/2002.

CHADA, J.S.; SARNO, L.; VALENTE, G. Monetary policy rules, asset prices, and exchange rates. IMF Staff Papers. v. 51, no 3, p. 529-552, 2004.

CLARIDA, R.; GALÍ, J.; GERTLER, M. Monetary policy rules in practice: some international evidence. European Economic Review, North-Holland, Elsevier Science Publishers B.V, v. 42, p. 1.033-1.067, 1998.

CLARIDA, R.; GALÍ, J.; GERTLER, M.. M. The science of monetary policy: a new keynesian perspective. NBER Working Paper, n 7.147, May/1999.

CLARIDA, R.; GALÍ, J.; GERTLER, M. The Science of monetary policy. Journal of Economic Literature, v. 37, n. 4, p. 1661-1707, December./1999.

COOPER, J.P.; FISCHER, S. Simulations of monetary rules in the FRB-MIT - penn model. Journal of Money. Credit and Banking, v. 4, n. 2, p. 384-396, May/1972.

COOPER, R. Exchange rate choices, in Jane Sneddon Little and Giovanni Olivei (Eds.). Rethinking the International Monetary System, Boston: Federal Reserve Bank of Boston, p. 99-123, 1999.

CUKIERMAN, A. Central Bank Strategy, credibility and independence: Theory and Evidence. Cambridge, Massachussets: MIT Press. 1992 p. 335-357

CUKIERMAN, A. Central Bank independence and monetary control. The Economic Journal, London, v.104, n.427, p. 1437 - 1448, November 1994.

ENGLE, R. F. Autoregressive conditional heteroscedasticity with estimates of the variance of United Kingdom inflation. Econometrica, v. 50, n. 4, p. 987-1007, July 1982.

FISCHER, S. Rules versus discretion in monetary policy. NBER Working Paper, n. 2.518, February 1988.

FRIEDMAN, M. A program for monetary stability. New York: Fordham University Press, 1960.

FRIEDMAN, M. The role of monetary policy. American Economic Review, Pittsburgh v. 58, p. 1-17, march 1968. 
JUDD, J.; RUDEBUSCH, G. Taylor's rule and the FED: 1970 - 1997. Economic Review. Federal Reserve Bank of San Francisco, v. 3, p. 3-16, 1998.

KYDLAND, F. E.; PRESCOTT, E. C. Rules rather than discretion: the inconsistency of optimal plans. Journal of Political Economic, Chicago, v. 85, n. 3, 1977.

LOHMANN, S. Optimal commitment in monetary policy: credibility versus flexibility. The American Economic Review. Pittsburgh, v.82, 1992.

LUCAS, R. E. Studies in business cycle theory. Cambridge: MIT Press, 1981. p .215 -39. v. 5.

MOLODTSOVA et al. Taylor rules and the euro. European area business cycle network conference. Economic Analysis, p.1-34, September, 2008. Disponível em: <www.ecb.int>. Acesso em: 16 nov. 2008.

OLIVEIRA, C.; CHUMVICHITRA, P. Credibilidade de regimes de câmbio fixo: uma evidência empírica da crise cambial brasileira. Teoria e Evidência Econômica. v. 13, n. 25, nov. 2005.

ORPHANIDES, Athanasios. Historical monetary Policy Analysis and the Taylor rule. Journal of Monetary Economics. v.50, n.5, p. 983-1022, July 2003.

ORPHANIDES, Athanasios Taylor rules. Board of Governors of the Federal Reserve System. January 2007. Disponível em: www.athanasiosorphanides.com/taylor22f.pdf. Acesso em: 16 nov. 2008.

POOLE, W. Monetary policy rules? Review Federal Reserve Bank of St. Louis. May/April 1999.

REINHART, Carmen M.; ROGOFF, Kenneth S. The modern history of exchange rate arrangements: a reinterpretation. NBER Working Paper, n. 8.963, Cambridge, June 2002.

ROGOFF, Kenneth. The optimal degree of commitment to an intermediate monetary target. The Quarterly Journal of Economics., The MIT Press, v. 100, n. 4, p. 1169 - 1189, November 1995.

TAYLOR, John B. Discretion versus policy rules in practice. Carnegie-Rochester Conference Series on Public Policy, n. 39, 1993.

TAYLOR, John B. Monetary policy rules. Chicago: University of Chicago, 1999.

TAYLOR, John B. The robustness and efficiency of monetary policy rules as guidelines for interest rate setting by the European Central Bank. Journal of Monetary Economics. v. 43, N3, July 1999.

TAYLOR, John B. The role of the exchange rate in monetary-policy rules. The American Economic Review, Pittsburgh .v. 91, n. 2. p. 263-267. May 2001.

TAYLOR, John B. Housing and Monetary Policy. In: Housing, Housing Finance, and Monetary Policy. Federal Reserve Bank of Kansas City, 2007.

TOBIN, James Inflation and unemployment. American Economic Review, Pittsburgh, v. 62, n.1, p.1-18, March 1972.

WALSH, Carl Optimal contracts for Central Bankers. American Economic Review, Pittsburgh v. 85, n. 1, 1995. 
ANEXO

Tabela 1 - Testes ADF com séries mensais para EUA, Japão e Área do euro

\begin{tabular}{|c|c|c|c|c|c|c|c|c|}
\hline & Nível & $\tau$ & $\tau_{\mu}$ & $\tau_{\tau}$ & $1^{0}$ diferença & $\tau$ & $\tau_{\mu}$ & $\tau_{\tau}$ \\
\hline \multirow{5}{*}{ 畕 } & $e_{t}$ & $-4,65^{*}$ & $-4,63^{*}$ & $-4,63^{*}$ & $e_{t}$ & $-11,84^{*}$ & $-11,81^{*}$ & $-11,78^{*}$ \\
\hline & $h_{t}$ & $-3,07^{*}$ & $-3,06^{*}$ & $-3,05(1)$ & $h_{t}$ & $-16,05^{*}$ & $-16,03^{*}$ & $-16,00^{*}$ \\
\hline & $\pi(6)_{t}$ & $-0,09(1)$ & $-2,77^{* *}$ & $-2,39(1)$ & $\pi(6)_{t}$ & $-9,54^{*}$ & $-9,52^{*}$ & $-9,79^{*}$ \\
\hline & $\pi(12)_{t}$ & $-0,18(1)$ & $-3,24^{*}$ & $-2,75(1)$ & $\pi(12)_{t}$ & $-5,18^{*}$ & $-5,16^{*}$ & $-5,64^{*}$ \\
\hline & $i_{t}$ & $-0,81(1)$ & $-3,02^{*}$ & $-4,67^{*}$ & $i_{t}$ & $-3,46^{*}$ & $-3,54^{*}$ & $-3,50^{*}$ \\
\hline \multirow{5}{*}{$\stackrel{0}{\Xi}$} & $e_{t}$ & $-4,58^{*}$ & $-4,57^{*}$ & $-4,56^{*}$ & $e_{t}$ & $-11,82^{*}$ & $-11,79^{*}$ & $-11,76^{*}$ \\
\hline & $h_{t}$ & $-2,27^{* *}$ & $-2,30(1)$ & $-2,37(1)$ & $h_{t}$ & $-4,31^{*}$ & $-4,30^{*}$ & $-4,35^{*}$ \\
\hline & $\pi(6)_{t}$ & $-0,10(1)$ & $-2,26(1)$ & $-1,94(1)$ & $\pi(6)_{t}$ & $-12,47^{*}$ & $-12,44^{*}$ & $-12,50^{*}$ \\
\hline & $\pi(12)_{t}$ & $-0,23(1)$ & $-2,47(1)$ & $-2,07(1)$ & $\pi(12)_{t}$ & $-14,86^{*}$ & $-14,83^{*}$ & $-14,95^{*}$ \\
\hline & $i_{t}$ & $-1,53(1)$ & $-3,69^{*}$ & $-2,52(1)$ & $i_{t}$ & $-3,08^{*}$ & $-3,43^{*}$ & $-4,66^{*}$ \\
\hline \multirow{5}{*}{ 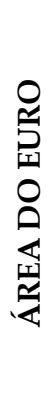 } & $e_{t}$ & $-3,50^{*}$ & $-3,48^{*}$ & $-3,48^{*}$ & $e_{t}$ & $-8,39^{*}$ & $-8,37^{*}$ & $-8,34^{*}$ \\
\hline & $h_{t}$ & $-2,44^{* *}$ & $-2,42(1)$ & $-2,37(1)$ & $h_{t}$ & $-11,78^{*}$ & $-11,76^{*}$ & $-11,73^{*}$ \\
\hline & $\pi(6)_{t}$ & $1,12(1)$ & $-1,31(1)$ & $-2,03(1)$ & $\pi(6)_{t}$ & $-14,29^{*}$ & $-14,35^{*}$ & $-14,38^{*}$ \\
\hline & $\pi(12)_{t}$ & $1,14(1)$ & $-0,96(1)$ & $-2,36(1)$ & $\pi(12)_{t}$ & $-9,33^{*}$ & $-9,38^{*}$ & $-9,39^{*}$ \\
\hline & $i_{t}$ & $0,35(1)$ & $-2,37(1)$ & $-2,20(1)$ & $i_{t}$ & $-2,09^{* * *}$ & $-2,09(1)$ & $-2,26(1)$ \\
\hline
\end{tabular}

Notas: $\tau$ sem constante; $\tau_{\mu}$ com constante; $\tau_{\tau}$ com constante e tendência; $\mathrm{H}_{0}$ : série possui raiz unitária (1); Rejeita Ho a $\left(^{*}: 1 \%\right),\left({ }^{* *}: 5 \%\right)$ ou $\left(^{* * *}: 10 \%\right)$; Seleção automática de defasagens - Critério de Schwartz. 
Tabela 2 - Resultados do teste Ng Perron para os EUA no período de janeiro/ 1990 a

\begin{tabular}{|c|c|c|c|c|c|c|c|c|c|}
\hline $\begin{array}{c}\text { Nível } \\
\tau_{\mu}\end{array}$ & MZa & $\mathbf{M Z t}$ & MSB & MPT & $\begin{array}{c}\text { Nível } \\
\tau_{\tau}\end{array}$ & MZa & $\mathbf{M Z t}$ & MSB & MPT \\
\hline$e_{t}$ & $\begin{array}{c}-35,46 \\
\left(^{*}\right)\end{array}$ & $\begin{array}{c}-3,57 \\
\left.{ }^{*}\right)\end{array}$ & $\begin{array}{c}0,13 \\
\left({ }^{*}\right)\end{array}$ & $\begin{array}{c}1,04 \\
(*)\end{array}$ & $e_{t}$ & $\begin{array}{c}-35,46 \\
\left(^{*}\right)\end{array}$ & $\begin{array}{c}-4,21 \\
\left(^{*}\right)\end{array}$ & $\begin{array}{c}0,11 \\
\left({ }^{*}\right)\end{array}$ & $\begin{array}{c}2,57 \\
\left(^{*}\right)\end{array}$ \\
\hline$h_{t}$ & $\begin{array}{c}-15,61 \\
\left(^{*}\right)\end{array}$ & $\begin{array}{c}-2,65 \\
\left(^{*}\right)\end{array}$ & $\begin{array}{c}0,16 \\
(*)\end{array}$ & $\begin{array}{l}2,10 \\
(*)\end{array}$ & $h_{t}$ & $\begin{array}{c}-17,50 \\
\left({ }^{* *}\right)\end{array}$ & $\begin{array}{c}-3,42 \\
\left({ }^{* *}\right)\end{array}$ & $\begin{array}{c}0,16 \\
\left(^{* *}\right)\end{array}$ & $\begin{array}{l}5,50 \\
(* *)\end{array}$ \\
\hline$\pi(6)_{t}$ & $\begin{array}{c}-0,88 \\
(1)\end{array}$ & $\begin{array}{c}-0,41 \\
(1)\end{array}$ & $\begin{array}{c}0,46 \\
(1)\end{array}$ & $\begin{array}{c}15,00 \\
(1)\end{array}$ & $\pi(6)_{t}$ & $\begin{array}{c}0,19 \\
(1)\end{array}$ & $\begin{array}{c}0,06 \\
(1)\end{array}$ & $\begin{array}{c}0,34 \\
(1)\end{array}$ & $\begin{array}{c}36,87 \\
(1)\end{array}$ \\
\hline$\pi(12)_{t}$ & $\begin{array}{c}-1,78 \\
(1)\end{array}$ & $\begin{array}{c}-0,94 \\
(1)\end{array}$ & $\begin{array}{c}0,52 \\
(1)\end{array}$ & $\begin{array}{c}13,69 \\
(1)\end{array}$ & $\pi(12)_{t}$ & $\begin{array}{c}-3,51 \\
(1)\end{array}$ & $\begin{array}{c}-1,03 \\
(1)\end{array}$ & $\begin{array}{c}0,29 \\
(1)\end{array}$ & $\begin{array}{c}21,42 \\
(1)\end{array}$ \\
\hline$i_{t}$ & $\begin{array}{c}-1,15 \\
(1)\end{array}$ & $\begin{array}{c}-0,56 \\
(1)\end{array}$ & $\begin{array}{c}0,49 \\
(1)\end{array}$ & $\begin{array}{c}14,99 \\
(1)\end{array}$ & $i_{t}$ & $\begin{array}{c}-91,23 \\
\left(^{*}\right)\end{array}$ & $\begin{array}{c}-6,75 \\
\left(^{*}\right)\end{array}$ & $\begin{array}{c}0,07 \\
(*)\end{array}$ & $\begin{array}{c}1,00 \\
\left({ }^{*}\right)\end{array}$ \\
\hline $\begin{array}{c}\mathbf{1}^{\mathbf{a}} \\
\text { diferença } \\
\mathrm{T} \mu \\
\end{array}$ & MZa & $\mathbf{M Z t}$ & MSB & MPT & $\begin{array}{c}1^{\mathbf{a}} \\
\text { diferença } \\
\tau_{\tau} \\
\end{array}$ & MZa & $\mathbf{M Z t}$ & MSB & MPT \\
\hline$e_{t}$ & $\begin{array}{c}-99,98 \\
\left(^{*}\right)\end{array}$ & $\begin{array}{c}-7,02 \\
\left(^{*}\right)\end{array}$ & $\begin{array}{c}0,07 \\
\left({ }^{*}\right)\end{array}$ & $\begin{array}{c}0,27 \\
(*)\end{array}$ & $e_{t}$ & $\begin{array}{c}-103,03 \\
\left(^{*}\right)\end{array}$ & $\begin{array}{c}-7,17 \\
\left(^{*}\right)\end{array}$ & $\begin{array}{c}0,06 \\
\left(^{*}\right)\end{array}$ & $\begin{array}{c}0,89 \\
(*)\end{array}$ \\
\hline$h_{t}$ & $\begin{array}{c}-11,06 \\
(* *)\end{array}$ & $\begin{array}{c}-2,27 \\
\left(^{*}\right)\end{array}$ & $\begin{array}{l}0,20 \\
(* *)\end{array}$ & $\begin{array}{l}2,50 \\
(*)\end{array}$ & $h_{t}$ & $\begin{array}{c}-35,96 \\
\left(^{*}\right)\end{array}$ & $\begin{array}{c}-4,24 \\
\left(^{*}\right)\end{array}$ & $\begin{array}{c}0,11 \\
\left(^{*}\right)\end{array}$ & $\begin{array}{c}2,53 \\
\left({ }^{*}\right)\end{array}$ \\
\hline$\pi(6)_{t}$ & $\begin{array}{c}-0,83 \\
(1)\end{array}$ & $\begin{array}{c}-0,39 \\
(1)\end{array}$ & $\begin{array}{c}0,46 \\
(1)\end{array}$ & $\begin{array}{c}15,10 \\
(1)\end{array}$ & $\pi(6)_{t}$ & $\begin{array}{c}-1,03 \\
(1)\end{array}$ & $\begin{array}{c}-0,39 \\
(1)\end{array}$ & $\begin{array}{c}0,37 \\
(1)\end{array}$ & $\begin{array}{c}36,26 \\
(1)\end{array}$ \\
\hline$\pi(12)_{t}$ & $\begin{array}{c}-15,31 \\
\left(^{*}\right)\end{array}$ & $\begin{array}{c}-2,58 \\
\left(^{*}\right)\end{array}$ & $\begin{array}{c}0,16 \\
\left({ }^{*}\right)\end{array}$ & $\begin{array}{c}1,78 \\
\left({ }^{*}\right)\end{array}$ & $\pi(12)_{t}$ & $\begin{array}{c}-227,41 \\
\left(^{*}\right)\end{array}$ & $\begin{array}{c}-10,62 \\
\left(^{*}\right)\end{array}$ & $\begin{array}{c}0,04 \\
\left(^{*}\right)\end{array}$ & $\begin{array}{c}0,51 \\
\left({ }^{*}\right)\end{array}$ \\
\hline$i_{t}$ & $\begin{array}{c}-20,64 \\
\left(^{*}\right)\end{array}$ & $\begin{array}{c}-3,13 \\
\left({ }^{*}\right)\end{array}$ & $\begin{array}{c}0,15 \\
\left({ }^{*}\right)\end{array}$ & $\begin{array}{c}1,46 \\
\left({ }^{*}\right)\end{array}$ & $i_{t}$ & $\begin{array}{c}-41,13 \\
\left({ }^{*}\right)\end{array}$ & $\begin{array}{c}-4,42 \\
\left(^{*}\right)\end{array}$ & $\begin{array}{c}0,10 \\
\left({ }^{*}\right)\end{array}$ & $\begin{array}{c}2,81 \\
\left(^{*}\right)\end{array}$ \\
\hline
\end{tabular}

Nota: $\tau_{\mu}$ com tendência; $\tau_{\tau}$ com constante e tendência; $\mathrm{H}_{0}$ : série possui raiz unitária; Rejeita $\mathrm{H}_{0}$ a (a: $1 \%$ ), (b: $5 \%$ ) ou (c: 10\%). Seleção automática de defasagens - Critério de Schwartz. 
Tabela 3 - Resultados do teste Ng Perron para o Japão no período de janeiro/ 1990 a junho/2008

\begin{tabular}{|c|c|c|c|c|c|c|c|c|c|}
\hline $\begin{array}{c}\text { Nível } \\
\tau_{\mu}\end{array}$ & $\mathrm{MZa}$ & $\mathbf{M Z t}$ & MSB & MPT & $\begin{array}{c}\text { Nível } \\
\tau_{\tau}\end{array}$ & $\mathrm{MZa}$ & $\mathbf{M Z t}$ & MSB & MPT \\
\hline$e_{t}$ & $\begin{array}{c}-21,21 \\
\left.{ }^{*}\right)\end{array}$ & $\begin{array}{c}-3,25 \\
\left.{ }^{*}\right)\end{array}$ & $\begin{array}{c}0,15 \\
\left(^{*}\right)\end{array}$ & $\begin{array}{c}1,17 \\
\left(^{*}\right)\end{array}$ & $e_{t}$ & $\begin{array}{c}-32,72 \\
\left(^{*}\right)\end{array}$ & $\begin{array}{c}-4,03 \\
\left(^{*}\right)\end{array}$ & $\begin{array}{c}0,12 \\
\left.{ }^{*}\right)\end{array}$ & $\begin{array}{c}2,86 \\
\left.{ }^{*}\right)\end{array}$ \\
\hline$h_{t}$ & $\begin{array}{c}19,05 \\
(1)\end{array}$ & $\begin{array}{c}16,38 \\
(1)\end{array}$ & $\begin{array}{c}0,85 \\
(1)\end{array}$ & $\begin{array}{c}239,44 \\
(1)\end{array}$ & $h_{t}$ & $\begin{array}{c}11,22 \\
(1)\end{array}$ & $\begin{array}{c}12,01 \\
(1)\end{array}$ & $\begin{array}{c}1,07 \\
(1)\end{array}$ & $\begin{array}{c}594,85 \\
(1)\end{array}$ \\
\hline$\pi(6)_{t}$ & $\begin{array}{c}-3,81 \\
(1)\end{array}$ & $\begin{array}{c}-1,36 \\
(1)\end{array}$ & $\begin{array}{c}0,35 \\
(1)\end{array}$ & $\begin{array}{c}6,42 \\
(1)\end{array}$ & $\pi(6)_{t}$ & $\begin{array}{c}-7,21 \\
(1)\end{array}$ & $\begin{array}{c}-1,68 \\
(1)\end{array}$ & $\begin{array}{c}0,23 \\
(1)\end{array}$ & $\begin{array}{c}13,00 \\
(1)\end{array}$ \\
\hline$\pi(12)_{t}$ & $\begin{array}{c}-2,13 \\
(1)\end{array}$ & $\begin{array}{c}-1,02 \\
(1)\end{array}$ & $\begin{array}{c}0,47 \\
(1)\end{array}$ & $\begin{array}{c}11,38 \\
(1)\end{array}$ & $\pi(12)_{t}$ & $\begin{array}{c}-7,61 \\
(1)\end{array}$ & $\begin{array}{c}-1,66 \\
(1)\end{array}$ & $\begin{array}{c}0,21 \\
(1)\end{array}$ & $\begin{array}{c}12,63 \\
(1)\end{array}$ \\
\hline$i_{t}$ & $\begin{array}{c}-0,34 \\
(1)\end{array}$ & $\begin{array}{c}-0,25 \\
(1)\end{array}$ & $\begin{array}{c}0,73 \\
(1)\end{array}$ & $\begin{array}{c}30,80 \\
(1)\end{array}$ & $i_{t}$ & $\begin{array}{c}-4,44 \\
(1)\end{array}$ & $\begin{array}{c}-1,35 \\
(1)\end{array}$ & $\begin{array}{c}0,30 \\
(1)\end{array}$ & $\begin{array}{c}19,46 \\
(1)\end{array}$ \\
\hline $\begin{array}{c}\mathbf{1}^{\mathbf{a}} \\
\text { diferença } \\
\tau \mu \\
\end{array}$ & $\mathrm{MZa}$ & $\mathbf{M Z t}$ & MSB & MPT & $\begin{array}{c}1^{\mathbf{a}} \\
\text { diferença } \\
\tau_{\tau} \\
\end{array}$ & $\mathrm{MZa}$ & $\mathbf{M Z t}$ & MSB & MPT \\
\hline$e_{t}$ & $\begin{array}{c}-2,30 \\
(1)\end{array}$ & $\begin{array}{c}-1,07 \\
(1)\end{array}$ & $\begin{array}{c}0,46 \\
(1)\end{array}$ & $\begin{array}{c}10,62 \\
(1)\end{array}$ & $e_{t}$ & $\begin{array}{c}-90,66 \\
\left(^{*}\right)\end{array}$ & $\begin{array}{c}-6,70 \\
\left(^{*}\right)\end{array}$ & $\begin{array}{c}0,07 \\
\left.{ }^{*}\right)\end{array}$ & $\begin{array}{c}1,10 \\
\left.{ }^{*}\right)\end{array}$ \\
\hline$h_{t}$ & $\begin{array}{c}-0,10 \\
(1)\end{array}$ & $\begin{array}{c}-0,22 \\
(1)\end{array}$ & $\begin{array}{c}2,06 \\
(1)\end{array}$ & $\begin{array}{c}210,28 \\
(1)\end{array}$ & $h_{t}$ & $\begin{array}{c}-0,30 \\
(1)\end{array}$ & $\begin{array}{c}-0,37 \\
(1)\end{array}$ & $\begin{array}{c}1,21 \\
(1)\end{array}$ & $\begin{array}{c}269,81 \\
(1)\end{array}$ \\
\hline$\pi(6)_{t}$ & $\begin{array}{c}-1,14 \\
(1)\end{array}$ & $\begin{array}{c}-0,75 \\
(1)\end{array}$ & $\begin{array}{c}0,65 \\
(1)\end{array}$ & $\begin{array}{c}21,03 \\
(1)\end{array}$ & $\pi(6)_{t}$ & $\begin{array}{c}-46,70 \\
\left(^{*}\right)\end{array}$ & $\begin{array}{c}-4,82 \\
\left(^{*}\right)\end{array}$ & $\begin{array}{c}0,10 \\
\left.{ }^{*}\right)\end{array}$ & $\begin{array}{c}2,00 \\
\left.{ }^{*}\right)\end{array}$ \\
\hline$\pi(12)_{t}$ & $\begin{array}{c}-284,1 \\
\left.{ }^{*}\right)\end{array}$ & $\begin{array}{c}-1191 \\
\left.{ }^{*}\right)\end{array}$ & $\begin{array}{c}0,04 \\
\left(^{*}\right)\end{array}$ & $\begin{array}{c}0,09 \\
\left.{ }^{*}\right)\end{array}$ & $\pi(12)_{t}$ & $\begin{array}{c}-9,22 \\
(1)\end{array}$ & $\begin{array}{c}-1,99 \\
(1)\end{array}$ & $\begin{array}{c}0,21 \\
(1)\end{array}$ & $\begin{array}{c}10,48 \\
(1)\end{array}$ \\
\hline$i_{t}$ & $\begin{array}{c}-2,32 \\
(1)\end{array}$ & $\begin{array}{c}-1,01 \\
(1)\end{array}$ & $\begin{array}{c}0,43 \\
(1)\end{array}$ & $\begin{array}{c}10,13 \\
(1)\end{array}$ & $i_{t}$ & $\begin{array}{c}-5,71 \\
(1)\end{array}$ & $\begin{array}{c}-1,66 \\
(1)\end{array}$ & $\begin{array}{c}0,29 \\
(1)\end{array}$ & $\begin{array}{c}15,91 \\
(1)\end{array}$ \\
\hline
\end{tabular}

Nota: $\tau_{\mu}$ com tendência; $\tau_{\tau}$ com constante e tendência; $\mathrm{H}_{0}$ : série possui raiz unitária; Rejeita $\mathrm{H}_{0} \mathrm{a}(\mathrm{a}: 1 \%),(\mathrm{b}: 5 \%)$ ou (c: 10\%). Seleção automática de defasagens - Critério de Schwartz. 
Tabela 4 - Resultados do teste Ng Perron para a Área do euro de janeiro/ 1990 a junho/ 2008

\begin{tabular}{|c|c|c|c|c|c|c|c|c|c|}
\hline $\begin{array}{c}\text { Nível } \\
\tau_{\mu}\end{array}$ & MZa & $\mathbf{M Z t}$ & MSB & MPT & $\begin{array}{c}\text { Nível } \\
\tau_{\tau}\end{array}$ & MZa & $\mathbf{M Z t}$ & MSB & MPT \\
\hline$e_{t}$ & $\begin{array}{l}-6,00 \\
(* * *)\end{array}$ & $\begin{array}{l}-1,59 \\
(* * *)\end{array}$ & $\begin{array}{l}0,26 \\
(* * *)\end{array}$ & $\begin{array}{l}4,50 \\
(* * *)\end{array}$ & $e_{t}$ & $\begin{array}{c}-13,08 \\
(1)\end{array}$ & $\begin{array}{l}-2,55 \\
(* * *)\end{array}$ & $\begin{array}{c}0,19 \\
(1)\end{array}$ & $\begin{array}{c}6,99 \\
(1)\end{array}$ \\
\hline$h_{t}$ & $\begin{array}{c}-5,06 \\
(1)\end{array}$ & $\begin{array}{c}-1,25 \\
(1)\end{array}$ & $\begin{array}{c}0,24 \\
(1)\end{array}$ & $\begin{array}{c}5,62 \\
(1)\end{array}$ & $h_{t}$ & $\begin{array}{c}-13,29 \\
(1)\end{array}$ & $\begin{array}{c}-2,48 \\
(1)\end{array}$ & $\begin{array}{c}0,18 \\
(1)\end{array}$ & $\begin{array}{c}7,40 \\
(1)\end{array}$ \\
\hline$\pi(6)_{t}$ & $\begin{array}{c}1,68 \\
(1)\end{array}$ & $\begin{array}{c}0,91 \\
(1)\end{array}$ & $\begin{array}{c}0,54 \\
(1)\end{array}$ & $\begin{array}{c}28,41 \\
(1)\end{array}$ & $\pi(6)_{t}$ & $\begin{array}{c}-6,70 \\
(1)\end{array}$ & $\begin{array}{c}-1,71 \\
(1)\end{array}$ & $\begin{array}{c}0,25 \\
(1)\end{array}$ & $\begin{array}{c}13,68 \\
(1)\end{array}$ \\
\hline$\pi(12)_{t}$ & $\begin{array}{c}0,06 \\
(1)\end{array}$ & $\begin{array}{c}0,01 \\
(1)\end{array}$ & $\begin{array}{c}0,31 \\
(1)\end{array}$ & $\begin{array}{c}11,82 \\
(1)\end{array}$ & $\pi(12)_{t}$ & $\begin{array}{c}-15,16 \\
\left({ }^{* * *}\right)\end{array}$ & $\begin{array}{l}-2,58 \\
(* * *)\end{array}$ & $\begin{array}{l}0,17 \\
\left(^{* * *}\right)\end{array}$ & $\begin{array}{c}6,97 \\
(1)\end{array}$ \\
\hline$i_{t}$ & $\begin{array}{c}-12,51 \\
(* *)\end{array}$ & $\begin{array}{c}-2,48 \\
\left({ }^{*}\right)\end{array}$ & $\begin{array}{c}0,19 \\
\left(^{* *}\right)\end{array}$ & $\begin{array}{c}2,00 \\
\left.{ }^{* *}\right)\end{array}$ & $i_{t}$ & $\begin{array}{c}-63,80 \\
\left(^{*}\right)\end{array}$ & $\begin{array}{c}-5,58 \\
\left(^{*}\right)\end{array}$ & $\begin{array}{c}0,08 \\
\left(^{*}\right)\end{array}$ & $\begin{array}{c}1,70 \\
\left(^{*}\right)\end{array}$ \\
\hline $\begin{array}{c}1^{\mathbf{a}} \\
\text { diferença } \\
\tau \mu \\
\end{array}$ & MZa & $\mathbf{M Z t}$ & MSB & MPT & $\begin{array}{c}\mathbf{1}^{\mathbf{a}} \\
\text { diferença } \\
\tau_{\tau} \\
\end{array}$ & $\mathrm{MZa}$ & $\mathbf{M Z t}$ & MSB & MPT \\
\hline$e_{t}$ & $\begin{array}{c}-56,12 \\
\left(^{*}\right)\end{array}$ & $\begin{array}{c}-5,29 \\
\left(^{*}\right)\end{array}$ & $\begin{array}{c}0,09 \\
\left({ }^{*}\right)\end{array}$ & $\begin{array}{c}0,43 \\
\left(^{*}\right)\end{array}$ & $e_{t}$ & $\begin{array}{c}-57,69 \\
\left(^{*}\right)\end{array}$ & $\begin{array}{c}-5,36 \\
\left(^{*}\right)\end{array}$ & $\begin{array}{c}0,09 \\
\left(^{*}\right)\end{array}$ & $\begin{array}{c}1,59 \\
\left(^{*}\right)\end{array}$ \\
\hline$h_{t}$ & $\begin{array}{c}-26,84 \\
\left({ }^{*}\right)\end{array}$ & $\begin{array}{c}-3,65 \\
\left(^{*}\right)\end{array}$ & $\begin{array}{c}0,13 \\
\left({ }^{*}\right)\end{array}$ & $\begin{array}{c}0,94 \\
\left({ }^{*}\right)\end{array}$ & $h_{t}$ & $\begin{array}{c}-80,49 \\
\left(^{*}\right)\end{array}$ & $\begin{array}{c}-6,33 \\
\left(^{*}\right)\end{array}$ & $\begin{array}{c}0,07 \\
\left(^{*}\right)\end{array}$ & $\begin{array}{c}1,15 \\
\left.{ }^{*}\right)\end{array}$ \\
\hline$\pi(6)_{t}$ & $\begin{array}{c}-4,95 \\
(1)\end{array}$ & $\begin{array}{c}-1,56 \\
(1)\end{array}$ & $\begin{array}{c}0,31 \\
(1)\end{array}$ & $\begin{array}{c}4,95 \\
(1)\end{array}$ & $\pi(6)_{t}$ & $\begin{array}{c}-4,70 \\
(1)\end{array}$ & $\begin{array}{c}-1,53 \\
(1)\end{array}$ & $\begin{array}{c}0,32 \\
(1)\end{array}$ & $\begin{array}{c}19,38 \\
(1)\end{array}$ \\
\hline$\pi(12)_{t}$ & $\begin{array}{c}-60,99 \\
\left(^{*}\right)\end{array}$ & $\begin{array}{c}-5,48 \\
\left({ }^{*}\right)\end{array}$ & $\begin{array}{c}0,08 \\
\left(^{*}\right)\end{array}$ & $\begin{array}{c}0,48 \\
\left({ }^{*}\right)\end{array}$ & $\pi(12)_{t}$ & $\begin{array}{c}-60,83 \\
\left({ }^{*}\right)\end{array}$ & $\begin{array}{c}-5,49 \\
\left(^{*}\right)\end{array}$ & $\begin{array}{c}0,09 \\
\left(^{*}\right)\end{array}$ & $\begin{array}{c}1,58 \\
\left(^{*}\right)\end{array}$ \\
\hline$i_{t}$ & $\begin{array}{c}-10,04 \\
(* *)\end{array}$ & $\begin{array}{c}-2,23 \\
\left({ }^{* *}\right)\end{array}$ & $\begin{array}{l}0,22 \\
\left({ }^{*}\right)\end{array}$ & $\begin{array}{c}2,44 \\
(* *)\end{array}$ & $i_{t}$ & $\begin{array}{c}-51,90 \\
\left(^{*}\right)\end{array}$ & $\begin{array}{c}-5,09 \\
\left(^{*}\right)\end{array}$ & $\begin{array}{c}0,09 \\
\left(^{*}\right)\end{array}$ & $\begin{array}{c}1,77 \\
\left(^{*}\right)\end{array}$ \\
\hline
\end{tabular}

Nota: $\tau_{\mu}$ com tendência; $\tau_{\tau}$ com constante e tendência; $\mathrm{H}_{0}$ : série possui raiz unitária; Rejeita $\mathrm{H}_{0}$ a (a: $1 \%$ ), (b: $5 \%$ ) ou (c: 10\%). Seleção automática de defasagens - Critério de Schwartz. 